\begin{tabular}{c|c|c}
\hline \hline Vol. 26: 149-157, 2017 & AQUATIC BIOLOGY \\
Aqtps://doi.org/10.3354/ab00680 & Aquat Biol & Published September 5 \\
\hline
\end{tabular}

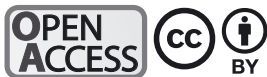

\section{Skin filament recovery after clipping in Hippocampus guttulatus: behavioural and histological aspects}

\author{
Michele Gristina ${ }^{1, *}$, Simona Bertrandino ${ }^{2}$, Frine Cardone ${ }^{2}$, Donatella Mentino ${ }^{2}$, \\ Giuseppe Corriero ${ }^{2}$, Giovanni Scillitani ${ }^{2}$
}

\author{
${ }^{1}$ CNR-IAMC, Via L. Vaccara, 61, 91142 Mazara del Vallo, TP, Italy \\ ${ }^{2}$ Dipartimento di Biologia, Università degli Studi di Bari, Via Orabona, 4, 70125 Bari, Italy
}

\begin{abstract}
Skin filaments are present on the heads of several Hippocampus species. Their clipping is a useful, non-lethal technique for marking individuals and sampling tissue. Little is known about the consequences of clipping on behaviour and details of regeneration. We present the results of a study on the effects of filament clipping on activity patterns and the regeneration of this structure at the macroscopic and microscopic level in Hippocampus guttulatus Cuvier, 1801 from the Gulf of Taranto (Apulia, Ionian Sea). Twelve individuals of both sexes underwent filament clipping, and their behaviour sequences (expressed as percent of total time spent swimming, resting, food searching, and swinging) were monitored for $10 \mathrm{wk}$ in water tanks. In the first week, individuals spent significantly more time food searching and swimming compared to controls, whereas in the following weeks, no differences between groups were observed. Regeneration was observed in 12 other individuals reclipped after 3, 5, and $7 \mathrm{wk}$. Sections of paraffin-embedded filaments were stained with Mallory's trichrome, alcian blue $\mathrm{pH} 2.5$, periodic acid-Schiff (PAS), and PAS with diastase. Epidermal cells and basal membranes were the first to recover fully. Basal epidermal cells were filled with glycogen. In the dermis, the arrangement of bundles of fibres surrounded by melanocytes took longer to reconstitute. After $10 \mathrm{wk}$, the filaments grew to about $36.7 \%$ of their original length, whereas at the microscopic level, the tissues recovered fully. It is concluded that filament clipping does not significantly affect the general behavioural patterns of $H$. guttulatus, and regeneration at the microscopic level is fully accomplished.
\end{abstract}

KEY WORDS: Fin clipping $\cdot$ Hippocampus guttulatus $\cdot$ Skin regeneration $\cdot$ Mediterranean Sea

\section{INTRODUCTION}

Marking is an essential technique in the study of fisheries biology, ecology, and aquaculture and has been widely used in a number of studies on migration patterns, growth rates, and the survival of breeding stocks (e.g. McKenzie et al. 2012). One of the most common marking methods is fin clipping, i.e. removal of a part of the fin. It is a non-lethal technique that requires minimal equipment, handling time, and training, and allows both marking and tissue sampling for population dynamics and genetic, pathological, and

${ }^{*}$ Corresponding author: michele.gristina@iamc.cnr.it ecotoxicological analysis (e.g. Feist et al. 2011, Jardine et al. 2011, Valladares \& Planas 2012, Becker et al. 2014, Monteiro et al. 2014, Petersson et al. 2014).

The introduction of non-lethal methods of tissue sampling allows researchers to study fish populations without affecting their numbers, removing resources from the ecosystem, or reducing a species' gene pool. Regarding conservation value, this technique permits the study of endangered and locally rare species, since it allows tissue sampling without lethal consequences for the organism. Seahorses of the genus Hippocampus are regarded as strongly threatened

() The authors 2017. Open Access under Creative Commons by Attribution Licence. Use, distribution and reproduction are unrestricted. Authors and original publication must be credited. 
by human impact, and therefore all species in the genus are included in the IUCN Red List Categories and Criteria (IUCN 2013).

In seahorses, fin clipping is broadly used to obtain tissues without exhibiting significant effects on their survival (Lourie 2003, Pardo et al. 2007). Additionally, seahorses have a high capacity for fin regeneration in 1 to 2 mo (Planas et al. 2008). Therefore, fin clipping may represent a useful, non-lethal sampling method to acquire seahorse tissues (Woodall et al. 2012). These animals are weak swimmers, with ear-like pectoral fins utilised for steering and a small dorsal fin mainly used for propulsion. Because of the importance of the dorsal fin, it is hard to predict what effect the removal of a portion would have on swimming capability. Several papers describe the high capacity for fin regeneration and low or null mortality rate due to this procedure; nonetheless, it could have effects on growth and survival rates (e.g. Murray \& Fuller 2000). However, most of the available data on fin clipping in seahorses were collected from captive animals, and the postoperative recovery was followed in aquaria, tanks, or mesocosms (Valladares \& Planas 2012). Thus, the fate of fin-clipped individuals in the natural environment is largely unknown and very difficult to assess.

In this context, skin filament clipping may be a nondetrimental alternative to fin-clipping; in fact several species of seahorses, including $H$. guttulatus, have thick skin filaments extending from spines on the head, superior trunk ridges, and neck that can be lost and then regenerated (Lourie et al. 2004) and are also capable of pattern-changing to blend in with the environment. The presence of skin filaments does not seem related to sex, size, life history stage, or reproductive status (Curtis \& Vincent 2006). Removal of these appendages to perform genetic analysis was described by Planas et al. (2008), but no detailed histological information about the recovery of fleshy filaments was reported. Furthermore, nothing is known about behavioural effects following filament clipping.

In the present study, we address the regeneration pattern after clipping of fleshy filaments using histological and histochemical techniques and examine possible alteration of behavioural sequences in the long-snouted seahorse $H$. guttulatus.

\section{MATERIALS AND METHODS}

\section{Collection and housing of animals}

A total of 36 adult individuals (15 males and 21 females) of Hippocampus guttulatus were randomly hand collected in the Mar Piccolo of Taranto (Apulia, Italy) in November 2015 at a mean depth of $0.5 \mathrm{~m}$. The collecting site was at Buffoluto, in a habitat characterised by an artificial hard substrate covered with brown algae (for more details, see Gristina et al. 2015 , 2017). All individuals were selected by appearance to ensure they possessed skin filaments and exhibited no external lesions or signs of unhealthy condition. These were sexed, weighed to the nearest $0.1 \mathrm{~g}$, measured for standard length (SL) to the nearest $\mathrm{mm}$, and then transferred to 1 of the 3 acclimatisation tanks (provided with 5001 of natural seawater and artificial holdfasts to normalise behaviour at $21.5 \pm 0.7^{\circ} \mathrm{C}$ ) in the laboratory of the University of Bari. None of the sampled males showed external signs of pregnancy. Throughout the experiment, the seahorses were fed 3 times a day with live (Gammarus aequicauda) and frozen (Artemia salina) food and housed under the natural photoperiod.

\section{Fleshy skin filament clipping procedure}

During the first $4 \mathrm{~d}$ after collection, individuals were acclimatised to the artificial conditions, and experimental investigations started when individuals began to feed regularly. Twenty-four individuals underwent the skin filament clipping procedure. Of these, 12 were used in the behavioural tests (Group 1), and 12 were used for the histological analysis of regrowth (Group 2). Twelve individuals with uncut filaments were considered as a control. To minimise the stress of clipping, the specimens were placed in small batches with a dilute clove oil solution (30 mg l $\mathrm{I}^{-1}$ ) for $4 \mathrm{~min}$ (Kroon 2015). Subsequently, the fleshy skin filament clipping procedure was carried out as follows: the operator, wearing aseptic gloves, held the seahorse with one hand and, using a toenail clipper, cut 3 to $4 \mathrm{~mm}$ of the skin filaments. The cut filament portions were rapidly fixed in $10 \%$ neutral buffered formalin for histological purposes.

After the cut (Fig. 1), to minimise behavioural differences, both clipped and control individuals were held in separate tanks (under the same conditions: 701 of natural seawater at $21.8 \pm 0.4^{\circ} \mathrm{C}$ ) and monitored daily for signs of disease and healing for a total of $10 \mathrm{wk}$.

\section{Behavioural patterns}

Experiments started in the last week of November 2015. Behavioural patterns (Aurelio et al. 2013) were 


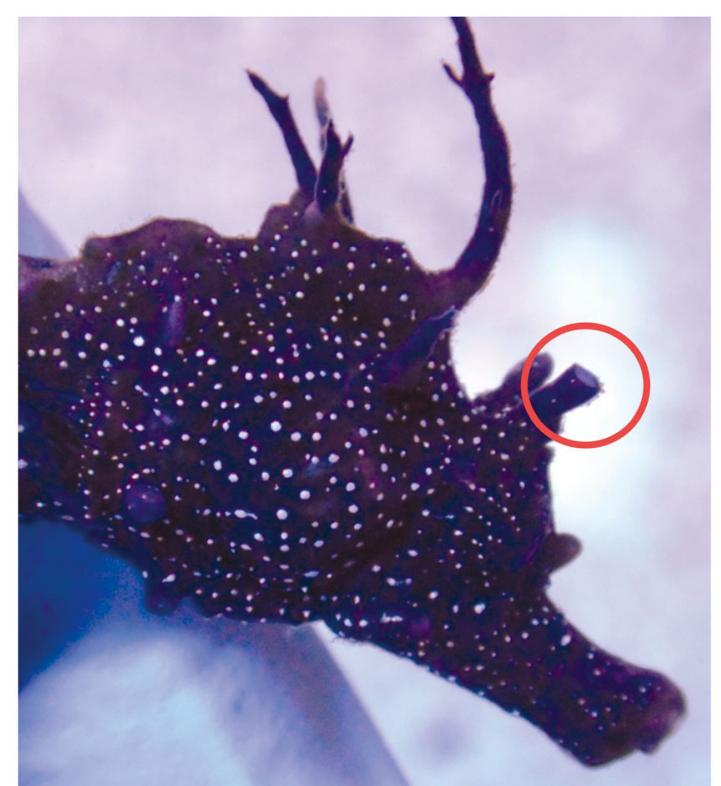

Fig. 1. Detail of skin filament cut in Hippocampus guttulatus

analysed for 24 individuals (12 control and 12 clipped). Observations were carried out for $10 \mathrm{wk}$ (November 2015 to January 2016). After the skin filament clipping, for the next $10 \mathrm{wk}$, a 60 min observation per week ( 30 min after 2 of the 3 feedings) was performed to assess any behavioural differences between the 2 treatments (control and clipped). To avoid interference due to the observer's presence, the behavioural observations were carried out by placing a black panel with small round holes in front of the aquaria. The behaviours of $H$. guttulatus were quantified as follows: (1) swimming (seahorse swims actively, moving the dorsal and pectoral fin); (2) resting (seahorse attached or unattached to the holdfast, remaining motionless over the substrate); (3) searching (seahorse tilts the body towards the aquarium floor in search of food); and (4) swinging (seahorse remains attached to the holdfast, with slight movements of the head or body). Behavioural patterns were expressed as a percentage of the time spent for each of these activities during the observation period.

Two-way distance-based permutational ANOVA (Anderson 2001) was performed to detect significant behavioural differences between clipped and control animals. This analysis, in which the $F$-statistics are calculated but $\mathrm{p}$-values are obtained by permutation, was performed rather than traditional univariate ANOVA, as it avoids any assumptions about the nature of the distribution of the original variables (Anderson 2001, Anderson \& ter Braak 2003). A 2way design was conducted, in which Treatment (Tr) was treated as a fixed factor with 2 levels (clipped and control) and Week (We) as a fixed and orthogonal factor with 10 levels (Week 1, Week 2, ... Week 10). Twenty-four replicates were collected for each combination of the factors. All analysis was based on the Euclidean distances in the original raw data, with all p-values obtained using 9999 permutations of the appropriate exchangeable units (Anderson \& ter Braak 2003). Significant terms in the full model were examined individually using appropriate a posteriori pairwise $t$-test comparisons, also conducted by permutations (Anderson 2001). The software applications PRIMER 6.1.10 and PERMANOVA+ b20 (www.primer-e.com) were used to perform all procedures.

\section{Macroscopic assessment of regrowth}

To assess skin filament regrowth, 12 specimens with clipped skin filaments (Group 1) were monitored weekly and photographed with a Samsung WP100 camera. The pictures were taken laterally to get a better shot of the cut filament edge. Photos were later analysed to determine the weekly percentage of regrowth (in length) using the tpsDig2 1.11 program. After the trials, all fish were released into the wild, approximately at the site of their capture.

\section{Histology}

For the microscopic analysis of regeneration patterns, the filaments from the first cut were compared with the regrown filaments from 4 individuals reclipped after 3, 5, and 7 wk (Group 2). The filaments were fixed in $10 \%$ neutral buffered formalin, dehydrated in a graded series of ethanol, and then embedded in paraffin wax. Sections were serially cut at $5 \mu \mathrm{m}$. The general morphology of tissues in the proximal area of the filament (i.e. near the cut) was assessed using Mallory's trichrome stain, whereas general and acidic glycans were detected using periodic acid-Schiff (PAS) and alcian blue (AB) pH 2.5 staining techniques, respectively (e.g. Scillitani et al. 2011, 2012). PAS staining experiments were also performed after a treatment with diastase to assess the presence of glycogen (Lillie \& Greco 1947). All the chemicals used are products of Sigma. Images were captured with a Nikon Eclipse 600 photomicroscope equipped with a Nikon DMX 1200 camera (Nikon Instruments, SpA). Staining in each experiment was assessed by at least 2 independent observers. 


\section{RESULTS}

The SL of the sampled animals was (mean \pm SD) $9.48 \pm$ $0.28 \mathrm{~cm}$ (min. 8.00, max. 11.00) in females and $9.62 \pm 0.41 \mathrm{~cm}$ (min. 7.80, max. 11.70) in males, while average weight was 3.32 $\pm 0.9 \mathrm{~g}$ (min. 2.04, max. 4.82) in females and $3.43 \pm 1.08 \mathrm{~g}$ (min. 2.01, max. 5.48) in males. No mortality occurred during the trial period.

\section{Behavioural patterns}

After the skin filament clipping procedure, individuals did not show any apparent symptoms of stress. The general behavioural patterns significantly changed during the trial period $(10 \mathrm{wk})$ for both treatments, which considered $\operatorname{Tr} \times$ We (2-way ANOVA; pseudo- $F_{9,220}=$ 3.14, p < 0.0001) (Table 1).

In the first $3 \mathrm{wk}$, active behaviours of both clipped and control individuals increased, reaching more than $45 \%$ of the monitored time. After Week 4 , we were able to observe a change in the general behavioural pattern, with an increase in swinging and resting behaviour. In the last $3 \mathrm{wk}$ (Weeks 8 to 10), this type of behaviour was more than $70 \%$ of the activities performed by the individuals under scrutiny (Fig. 2).

Pairwise $t$-tests performed for the interaction term $\operatorname{Tr} \times$ We showed significant differences only in the first 2 wk of monitoring ( $<<0.001$; Table 1), while in the following weeks, this test revealed non-significant differences in the behavioural pattern of clipped and control animals $(p>0.1)$ (Table 1).

\section{Macroscopic assessment of regrowth}

Photographic monitoring (Fig. 3), performed to assess regrowth of skin filaments, showed a mean weekly regrowth rate of (mean $\pm \mathrm{SD}$ ) $3.37 \pm 1.27 \%$, which, after $10 \mathrm{wk}$ of observations, represented around $37.6 \pm 3.36 \%$ ) of the skin filament clipped portion. No differences were observed between sexes.

\section{Histology}

The integument of filaments from the first cut presented an epidermis consisting of 2 cell layers (Fig. 4A). Epithelial cells were cuboid in shape, with a euchromatic nucleus and an evident nucleolus. The apical surface of the outer cells was ornamented with microridges (Fig. 4A). Interspersed among the epithelial cells were flame cone cells, with their apical parts protruding from the surface (Fig. 4A); club cells with unstained contents (Fig. 4A); and a few melanophores (Fig. 4I). Epithelial and flame cone cells were faintly stained with PAS (Fig. 4B). No multicellular receptors, such as taste buds, were observed. The epithelial cells of the basal layer were separated from the underlying dermis by a basement membrane, which was stained blue with both $A B$ pH 2.5 (Fig. 4C) and Mallory's trichrome (Fig. 4A) and red with PAS (Fig. 4B). The dermis consisted of a dense connective tissue in which the branches of the underlying melanophores insinuated, dividing it into a series of bundles of densely packed fibres running parallel to the integument surface (Fig. 4A). The melanophores formed a layer interposed between the dermis and the hypodermis, which, at some points, was crossed by the bundles of connective fibres fastening the dermis to the hypodermis. The hypodermis was made of a loose areolar connec- 


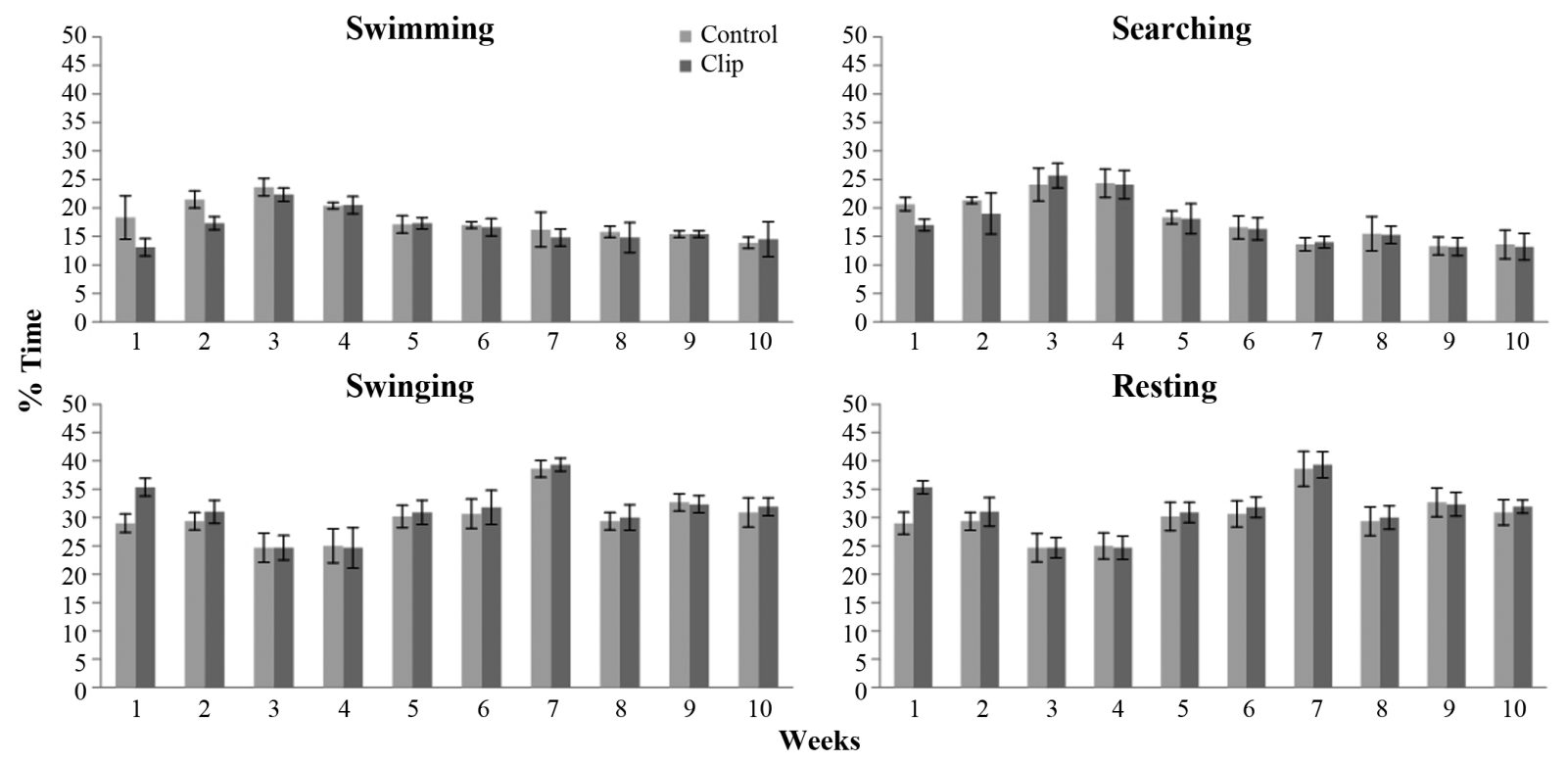

Fig. 2. Temporal trend of the behavioural pattern of clipped $(\mathrm{n}=12)$ and control $(\mathrm{n}=12)$ Hippocampus guttulatus individuals. Data are mean $\pm \mathrm{SD}$

tive tissue with large lacunae surrounded by bundles of connective fibres without a precise orientation (Fig. 4A). In the hypodermis, sparse fibrocytes, melanophores, and small blood vessels were detected (Fig. 4A,C). No scales were observed. Both the dermis and the hypodermis were stained faintly with PAS (Fig. 4B) and strongly with both Mallory's trichrome and $\mathrm{AB}$ pH 2.5 (Fig. 4A, C).

Three weeks after clipping, the regenerating filament appeared entirely reconstituted, with cells arranged in 2 to 5 layers (Fig. 4D). The cells in the basal layer were partially filled with a strongly PASpositive material that disappeared after diastase pretreatment (Fig. 4E). Flame cone cells and club cells were also observed (Fig. 4E). The basement membrane was reconstituted as well and stained intensely with PAS (Fig. 4E). In the dermis, the fibres were arranged more irregularly than previously, so the parallel bundles were not evident (Fig. 4D). A few melanophores appeared and showed an ovular rather than a branched shape (Fig. $4 \mathrm{D}, \mathrm{E}, \mathrm{F}$ ). In the hypodermis, the connective fibres appeared more abundant but less densely packed than previously, and some of them ran towards the dermis (Fig. 4D). Blood vessels appeared entirely reconstituted. Five weeks after clipping, in the epidermis, the epidermal cells of a basal layer still had a PAS-positive cytoplasm that became PAS negative after diastase treatment (Fig. 4H). The dermis layer was more well defined, with the presence of bundles that had become more densely packed (Fig. 4G). Melanophores increased in number in the underlying layer, and their shape changed from ovular to branched (Fig. 4G). In the hypodermis, the bundles of connective fibres appeared more densely packed (Fig. 4G). Seven wk after clipping, morphology and histochemical staining of the filament were very similar to the original condition (not shown). No differences were observed between sexes.

\section{DISCUSSION}

As already suggested by Lourie et al. (2004), skin filaments seem to be plastic appendages that may change colour and regrow in a short period and may assume a different shape from the original. The present study demonstrated no significant consequences to Hippocampus guttulatus after skin filament clipping. After $10 \mathrm{wk}$, the regeneration of skin filaments was about $37.6 \%$, which appears lower than that observed for the regrowth of the dorsal fin by Planas et al. (2008) for $H$. guttulatus and by Woodall et al. (2012) for H. kuda (4 to $6 \mathrm{wk}$ ).

\section{Behavioural patterns}

H. guttulatus individuals subjected to the tank conditions showed a significant change in behaviour during the $10 \mathrm{wk}$ of the trial (Table 1). At the beginning of the trial, all monitored individuals exhibited 


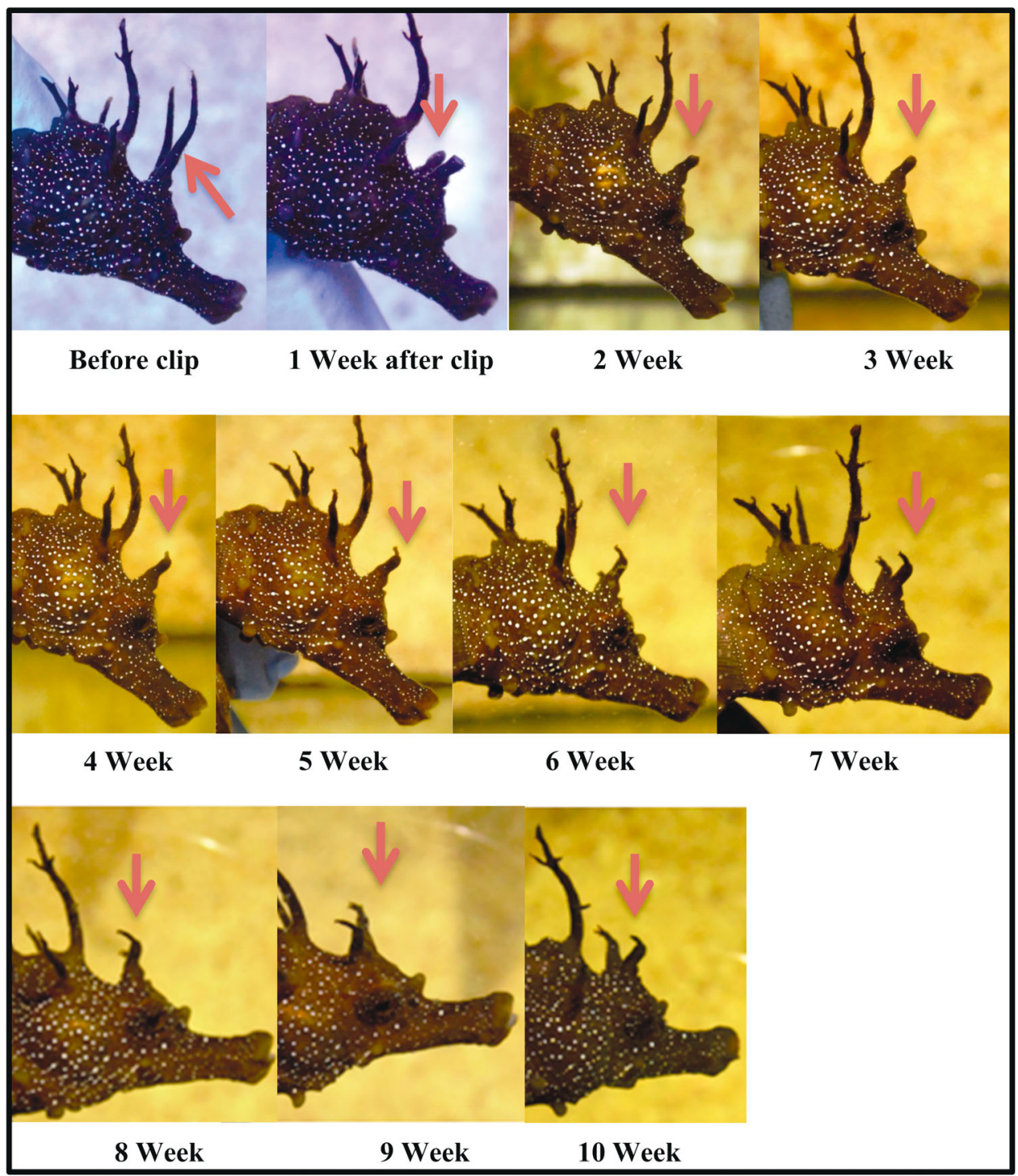

Fig. 3. Photo sequence of skin filament cut and regrowth in Hippocampus guttulatus

intense swimming activity and were looking for prey more than $50 \%$ of the time. After the third week of observation, the general behavioural pattern of both treatments (clipped and control) changed, and animals preferred to remain sedentary, attached to the holdfast and resting or swinging. In this phase, the activity patterns of $H$. guttulatus were similar to those observed by Aurelio et al. (2013) in the same species from the Atlantic coast of Portugal. These authors found that the animals maintained the same relative activity times despite changing water temperatures. The cutting of the skin filament in our sample apparently affected the activity patterns in only the first 2 wk; afterwards, the animals recovered, and their ethograms returned to patterns similar to those of the controls.

\section{Histology}

Literature data about the microscopic anatomy of the skin of seahorses are scarce (Linton \& Soloff 1964, Bereiter-Hahn et al. 1980, Zaccone \& Licata 1982, Carcupino et al. 2002, Oconer et al. 2003), and many deal with the skin of the breeding pouch of males. Linton \& Soloff (1964) found PAS-reactive material in the basal epithelial cells of the pouch in the nonbreeding condition and interpreted it as glycogen. The PAS negativity following diastase pretreatment in our experiments confirms that the epithelial basal cells contain glycogen, probably required for the increased metabolic activities linked to growth and division. The euchromatic nuclei also denote an active proliferation, and therefore it is not surprising 


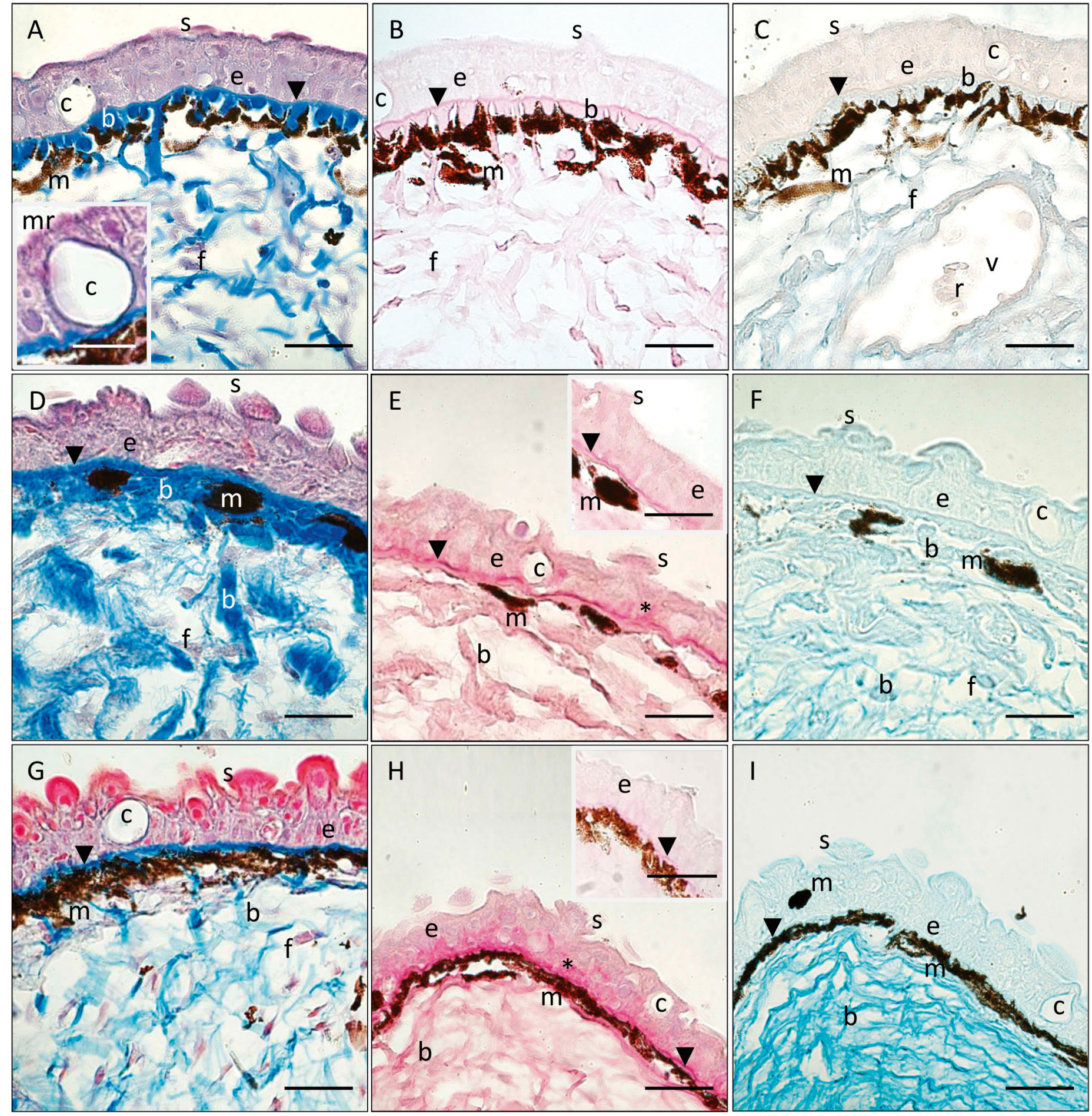

Fig. 4. Transverse sections of native and regenerating filament in Hippocampus guttulatus. (A,B,C) Native filament. Inset in (A) shows details of the apical surface of epitheliocytes with microridges. (D,E,F) Regenerating filament after 3 wk. Inset in (E) shows the same stain after diastase treatment, indicating disappearance of periodic acid-Schiff (PAS)-positive material in the basal cells. $(\mathrm{G}, \mathrm{H}, \mathrm{I})$ Regenerating filament after $5 \mathrm{wk}$. Inset in $(\mathrm{H})$ shows the same stain after diastase treatment, indicating disappearance of PAS-positive material in the basal cells. Stains: Mallory's trichrome in $(A, D, G) ; P A S$ in $(B, E, H)$; alcian blue pH 2.5 in $(\mathrm{C}, \mathrm{F}, \mathrm{I})$. All scale bars $=20 \mu \mathrm{m}$ except for inset in $(\mathrm{A})=10 \mu \mathrm{m}$. b: bundle of collagen fibres; c: club cell; e: epithelial cell; f: fibrocyte; m: melanophore; mr: microridge; $r$ : red blood cell; $s$ : epithelial cell in shedding; v: blood vessel; arrowhead: basement membrane; asterisk: glycogen in basal cell 
that the complete recovery of epithelium in our samples was faster than that of the underlying connective tissue. Besides, in the course of wound healing, epidermal cells move towards the injured areas as compact groups, without losing the normal intercellular junctions (Iger \& Abraham 1990), so that the general morphology of the epithelium is not altered in the process. The flame cone cells also showed a similar rate of regeneration. These cells are named for their peculiar shape and are unique to Hippocampus species (Bereiter-Hahn et al. 1980). Both epithelial and flame cone cells secrete mucins (Zaccone \& Licata 1982). The mucus cap produced by the latter has the function of maintaining the proliferation of epiphytes and increase the density of the skin's extra-cellular matrix (Bereiter-Hahn et al. 1980). Secretion is difficult to see with standard histochemical techniques like those we used, since it appears as a very thin layer at the surface of cells (Bereiter-Hahn et al. 1980). The function of club cells is obscure (Chivers et al. 2007). Ralphs \& Benjamin (1992) hypothesised that these cells have a role in wound healing, although we did not notice any change in their number during the process of regeneration.

The dermis exhibits parallel bundles of fibres that have a role in supporting filaments, since no scales are present. A similar arrangement is seen in nakedskin species, such as a number of catfishes (Le Guellec et al. 2004). The branched arrangement of melanophores in the dermis probably allows the translocation of melanosomes throughout the cells in the process of physiological colour change (e.g. Nilsson Sköld et al. 2013). Recovery of the dermis and hypodermis to the original condition required a longer time compared to the epidermis, since the reorganisation of cells and fibres is more complex than that of the epithelium (Iger \& Abraham 1990). Fibroblasts first exhibit phagocytic activity to remove debris from the wounded area and only subsequently start the secretion of collagen fibres (Iger \& Abraham 1990). These in turn are arranged in bundles following the reorganisation of the connective tissues. Melanophores are known to have both regenerating and migrating properties in wound healing. When these cells migrate, they have an ovoidal shape and exhibit amoeboid movements (e.g. Takahashi \& Kondo 2008). We can hypothesise that the ovoid melanophores we observed in the regenerating filament were migrating from other areas and that they subsequently returned to the branched shape after the reorganisation of the bundles of fibres. On the basis of our observations, the filament does not seem to have a particularly important function in protec- tion or sensation, and the area of the cut regenerates fully after about $7 \mathrm{wk}$.

Although the findings of several laboratories support fin clipping as a safe and efficient method for marking and tissue sampling, it may have adverse effects on a variety of behaviours, including swimming performance, prey capture, and predator avoidance (e.g. see the review of Eriksen et al. 2011).

For small endangered fish taxa, especially seahorses, it has been demonstrated that the fin-clipping method has some drawbacks compared to fleshy skin filament clipping because seahorses (and other fishes) usually contract their fin over the body during handling, thus requiring longer manipulation times (Planas et al. 2008). Unlike fin clipping, which cuts both skin and fin rays, skin filament clipping allows cutting epidermal and non-ossified dermal tissues exclusively, potentially reducing the pain induced (since no evident sensorial structures are present), the effects on swimming performance, and the regeneration time (Morgan \& Bull 2005). Ideal laboratory conditions may positively affect the survival and swimming performance of fishes that have been subjected to fin clipping, although the risk of infection is low after this procedure (Woodall et al. 2012). Additional histological and behavioural studies on the effects of skin filament clipping in small fishes with high camouflage capacity should be conducted in the field to evaluate survival under natural conditions.

Acknowledgements. This study was carried out under project and personnel licenses granted by the Italian Ministry of the Environment and Protection of Land and Sea (Prot no. 0021033/18.09.2013)

\section{LITERATURE CITED}

Anderson MJ (2001) A new method for non-parametric multivariate analysis of variance. Austral Ecol 26:32-46

Anderson MJ, ter Braak CJF (2003) Permutation test for multi-factorial analysis of variance. J Stat Comput Simul 73:85-113

Aurelio M, Faleiro F, Lopes VM, Pires V and others (2013) Physiological and behavioural responses of temperate seahorses (Hippocampus guttulatus) to environmental warming. Mar Biol 160:2663-2670

*Becker LA, Kirkland M, Heath JW, Heath DD, Dixon B (2014) Breeding strategy and rearing environment effects on the disease resistance of cultured Chinook salmon (Oncorhynchus tshawytscha). Aquaculture 422-423: 160-166

Bereiter-Hahn J, Richards K, Elsner L, Voth M (1980). Composition and formation of flame cell caps: a substratum for the attachment of micro-organisms to sea horse epidermis. Proc R Soc Edinb Biol 79:105-112

Carcupino M, Baldacci A, Mazzini M, Franzoi P (2002) Functional significance of the male brood pouch in the repro- 
ductive strategies of pipefishes and seahorses: a morphological and ultrastructural comparative study on three anatomically different pouches. J Fish Biol 61:1465-1480

Chivers DP, Wisenden BD, Hindman CJ, Michalak TA and others (2007) Epidermal 'alarm substance' cells of fishes maintained by non-alarm functions: possible defence against pathogens, parasites and UVB radiation. Proc $\mathrm{R}$ Soc B 274:2611-2619

* Curtis JMR, Vincent ACJ (2006) Life history of an unusual marine fish: survival, growth and movement patterns of the European long-snouted seahorse, Hippocampus guttulatus (Cuvier 1829). J Fish Biol 68:707-733

Eriksen TB, Fraser T, Gregersen H, Kristiansen $M$ and others (2011) Should fin clipping be used as a method for identification of fish? Norecopa, Oslo

Feist BE, Buhle ER, Arnold P, Davis JW, Scholz NL (2011) Landscape ecotoxicology of coho salmon spawner mortality in urban streams. PLOS ONE 6:e23424

Gristina M, Cardone F, Carlucci R, Castellano L, Passarelli S, Corriero G (2015) Abundance, distribution and habitat preference of Hippocampus guttulatus and Hippocampus hippocampus in a semi-enclosed central Mediterranean marine area. Mar Ecol 36:57-66

* Gristina M, Cardone F, Desiderato A, Mucciolo S, Lazic T, Corriero G (2017) Habitat use in juvenile and adult life stages of the sedentary fish Hippocampus guttulatus. Hydrobiologia 784:9-19

Iger Y, Abraham M (1990) The process of skin healing in experimentally wounded carp. J Fish Biol 36:421-437

IUCN (2013) IUCN Red List of Threatened Species. www. iucnredlist.org/ (Accessed 15 Jan 2014)

* Jardine TD, Hunt RJ, Pusey BJ, Bunn SE (2011) A non-lethal sampling method for stable carbon and nitrogen isotope studies of tropical fishes. Mar Freshw Res 62:83-90

Kroon FJ (2015) The efficacy of clove oil for anaesthesia of eight species of Australian tropical freshwater teleosts. Limnol Oceanogr Methods 13:463-475

Le Guellec D, Morvan-Dubois G, Sire JY (2004) Skin development in bony fish with particular emphasis on collagen deposition in the dermis of the zebrafish (Danio rerio). Int J Dev Biol 48:217-231

Lillie RD, Greco J (1947) Malt diastase and ptyalin in place of saliva in the identification of glycogen. Stain Technol 22:67-70

Linton JR, Soloff BL (1964) The physiology of the brood pouch of the male sea horse Hippocampus erectus. Bull Mar Sci 14:45-61

Lourie S (2003) Fin-clipping procedure for seahorses. Project Seahorse Technical Bulletin No. 3, Version 1.1. Project Seahorse, University of British Columbia, Vancouver

Lourie SA, Foster SJ, Cooper EWT, Vincent ACJ (2004) A guide to the identification of seahorses. Project Seahorse and TRAFFIC North America. University of British Columbia and World Wildlife Fund, Washington, DC

McKenzie JR, Parsons B, Seitz AC, Kopf RK, Mesa M, Phelps $\mathrm{Q}$ (eds) (2012) Advances in fish tagging and marking technology. American Fisheries Society, Symposium 76, Bethesda, MD

Monteiro NM, Silva RM, Cunha M, Antunes A, Jones AG, Vieira MN (2014) Validating the use of colouration pat- terns for individual recognition in the worm pipefish using a novel set of microsatellite markers. Mol Ecol Resour 14:150-156

Morgan S, Bull C (2005) Potential techniques for tagging and marking seahorses. Project Seahorse Technical Report No.7, Version 1.0. Project Seahorse, University of British Columbia, Vancouver

Murray DL, Fuller MR (2000) A critical review of the effects of marking on the biology of vertebrates. In: Boitani L, Fuller TK (eds) Research techniques in animal ecology: controversies and consequences. Columbia University Press, New York, NY, p 15-64

Nilsson Sköld H, Aspengren S, Wallin M (2013) Rapid color change in fish and amphibians-function, regulation, and emerging applications. Pigment Cell Melanoma Res 26: 29-38

Oconer E, Amparado E, Kime D (2003) Reproductive morphology and gonad development of the male seahorse, Hippocampus barbouri Jordan and Richardson 1908. Asia Life Sci 12:27-38

* Pardo BG, López A, Martínez P, Bouza C (2007) Novel microsatellite loci in the threatened European longsnouted seahorse (Hippocampus guttulatus) for genetic diversity and parentage analysis. Conserv Genet 8: 1243-1245

*Petersson E, Rask J, Ragnarsson B, Karlsson L, Persson J (2014) Effects of fin-clipping regarding adult return rates in hatchery-reared brown trout. Aquaculture 422-423: 249-252

Planas M, Chamorro A, Quintas P, Vilar A (2008) Establishment and maintenance of threatened long-snouted seahorse, Hippocampus guttulatus, broodstock in captivity. Aquaculture 283:19-28

Kalphs JR, Benjamin M (1992) Chondroitin and keratan sulphate in the epidermal club cells of teleosts. J Fish Biol 40:473-475

* Scillitani G, Moramarco AM, Rossi R, Mastrodonato M (2011) Glycopattern analysis and structure of the egg extra-cellular matrix in the Apennine yellow-bellied toad, Bombina pachypus (Anura: Bombinatoridae). Folia Histochem Cytobiol 49:306-316

Scillitani G, Mentino D, Liquori GE, Ferri D (2012) Histochemical characterization of the mucins of the alimentary tract of the grass snake, Natrix natrix (Colubridae). Tissue Cell 44:288-295

Takahashi G, Kondo S (2008.) Melanophores in the stripes of adult zebrafish do not have the nature to gather, but disperse when they have the space to move. Pigment Cell Melanoma Res 21:677-686

*Valladares S, Planas M (2012) Non-lethal dorsal fin sampling for stable isotope analysis in seahorses. Aquat Ecol 46:363-370

* Woodall LC, Jones R, Zimmerman B, Guillaume S, Stubbington T, Shaw P, Koldewey HJ (2012) Partial fin-clipping as an effective tool for tissue sampling seahorses, Hippocampus spp. J Mar Biol Assoc UK 92:1427-1432

Zaccone G, Licata A (1982) Histochemistry and finestructure of the flame cone cells in the skin epidermis of the sea horse fish Hippocampus ramulosus Leach 1814 (Teleostei, Syngnathidae). Arch Biol (Liege) 93:249-266

Submitted: February 7, 2017; Accepted: June 23, 2017

Proofs received from author(s): August 29, 2017
Editorial responsibility: Victor Benno Meyer-Rochow, Oulu, Finland 